\title{
Tangence
}

\section{Quelques garanties pour une lecture littéraire}

\section{Louise Milot}

Numéro 36, mai 1992

La lecture littéraire

URI : https://id.erudit.org/iderudit/025709ar

DOI : https://doi.org/10.7202/025709ar

Aller au sommaire du numéro

Éditeur(s)

Tangence

ISSN

0226-9554 (imprimé)

1710-0305 (numérique)

Découvrir la revue

Citer cet article

Milot, L. (1992). Quelques garanties pour une lecture littéraire. Tangence, (36), 34-40. https://doi.org/10.7202/025709ar d'utilisation que vous pouvez consulter en ligne.

https://apropos.erudit.org/fr/usagers/politique-dutilisation/ 


\section{Quelques garanties pour une lecture littéraire}

\section{Louise Milot}

En promettant des garanties, même si ce n'est que quelquesunes, mon titre peut paraître résolument optimiste. À certains moments, quand on chemine dans une recherche, on croit forcément au progrès, on croit avoir trouvé quelque chose: l'optimisme n'est que cela.

Il est vrai tout de même que depuis trois ou quatre ans que j'anime, au CRELIQ de l'Université Laval, une recherche sur ce que nous avons d'abord appelé l'inscription du littéraire, puis l'inscription des figures du littéraire, et finalement "L'inscription des figures de l'écrit dans le roman québécois", j'ai l'impression de toucher quelque chose qui aurait à voir avec le phénomène textuel du littéraire - dans le contexte romanesque tout au moins - et de tenir avec cette approche une manière de saisir avec quelque pertinence ce qui en constituerait une caractéristique importante, même si ce ne devait pas en être la seule. Mon propos ici n'est pas d'aborder de front cette problématique. Je concède d'ailleurs que la question de la possibilité ou non de trancher à savoir si un texte, au-delà des lectures particulières qu'on pourrait en faire, est ou n'est pas littéraire, nécessiterait au moins un débat qu'il n'y a pas lieu de susciter ici. Ayant par ailleurs essayé de le faire lors d'un colloque sur la question en $1989^{1}$, je sais qu'un tel débat n'est pas facile et qu'il est forcément passionné.

Ce que je veux indiquer simplement est qu'à travers les recherches auxquelles je viens de faire allusion, jamais jusqu'ici notre équipe n'a posé le problème en termes de lecture. La raison en est assez simple; se placer du point de vue de la lecture, pour nous, c'eût été pactiser ou avec les sociologies de la littérature, ou, plus précisément, avec les esthétiques de la réception. Or, dans la mesure où la recherche privilégiait - dans un premier volet tout au moins - la détermination de critères textuels de

1 Voir Louise Milot et Fernand Roy (éd.), La littérarité, Québec, PUL, 1991. Actes d'un colloque tenu en novembre 1989. 
littérarité, elle se devait, nous semblait-il, de se tenir éloignée, du même coup, des unes comme des autres.

C'est donc la première fois - on l'aura deviné par l'accumulation de précautions qui précède - que j'aborde directement la question de la lecture ${ }^{2}$; la première fois que je conviens de me placer du point de vue, non pas d'une éventuelle littérarité des textes, mais du caractère éventuellement littéraire de leur lecture. Peut-être que ce que notre équipe essaie d'élucider mène aussi à la proposition d'une sorte de lecture qu'il faudra bien appeler "littéraire". À moins que les deux - le texte littéraire et la lecture littéraire - reviennent au même, comme l'envers et l'endroit d'une même feuille de papier, pour reprendre une image connue.

Je ne le crois pas pourtant; ou tout au moins je ne l'affirmerais pas sans faire un détour. Et j'en viens par là à l'état présent de ma réflexion sur la question que je veux très simplement vous soumettre pour l'interroger: y a-t-il une différence de nature entre ce que serait, par exemple, la spécificité littéraire d'un texte, et ce que serait une lecture soi-disant littéraire qu'on pourrait en faire? En d'autres mots, et de même qu'on peut lire un texte littéraire de plusieurs manières dont la plupart ont peu à voir avec la littérature, pourrait-on lire littérairement un texte non littéraire? Ce qui impliquerait, je le signale, que si une culture donnée comporte des textes "constitutivement " 3 littéraires, (Phèdre de Racine, par exemple), elle comporterait aussi des textes "constitutivement" non littéraires; par le fait même, n'importe quel texte ne pourrait pas devenir littéraire du seul fait d'une décision de lecture, contrairement à ce que propose Gérard Genette dans Fiction et diction.

Le premier chapitre de cet ouvrage, où Genette aborde de front pour la première fois, sauf erreur, la question de la littérarité, l'amène au bout d'une assez longue démonstration à retenir la catégorie de l'intransitivité comme celle qui ultimement "constitue le texte en objet autonome et sa relation au lecteur en relation

2 J'ai d'ailleurs recommencé au colloque *Traditions de lecture - tenu au CRELIQ de l'Université Laval en novembre 1991, dans * La lecture et ses contextes: de Jean Chrétien à Charles Baudelaire (à paraître) et qui a en commun avec le présent texte d'avoir pour point de départ les positions récentes de Genette.

3 L'expression est de Genette dans Fiction et diction (Paris, Seuil, 1990) où il oppose les *littérarités constitutives * aux * littérarités conditionnelles * 
esthétique" (p. 37). Pour lui, non seulement tout texte de fiction est intransitif, donc littéraire, et appelle d'être lu comme tel du fait de la stratégie fictive même, mais n'importe quel texte de nonfiction est susceptible de devenir littéraire, si et seulement si, je dirais, "un jugement de goût individuel ou collectif [une lecture donc] [...] fait passer au premier plan ses qualités esthétiques" (p. 28). Il me semble pouvoir déduire de cette position qu'il n'y a pas en principe de limite, pour Genette, à l'extension des textes littéraires, ni de différence d'extension entre les textes littéraires et les lectures littéraires des textes, tout au moins pour les textes non encore institutionnalisés ${ }^{4}$. La possibilité qu'un texte soit lu littérairement est renvoyée au lecteur, dont le pouvoir de rendre intransitif un texte est alors très large, puisque la lecture pourrait toujours infléchir vers la fonction esthétique "tout texte dont la fonction originelle n'était pas celle-là " (p. 28). Dire que je ne suis pas encline à suivre Genette sur ce terrain ne signifie pas, loin de là, que la question soit simple, ni que j'aie moi-même les moyens de l'élucider.

Pour mettre les choses en perspective, je prendrai le cas d'un autre type de discours, comparable au discours littéraire - il s'agit souvent d'une procédure éclairante: le discours scientifique. Et je reviendrai sur deux commentaires d'A.J. Greimas à propos de ce discours; l'un date de 1976, dans "Du discours scientifique en sciences sociales" 5 , l'autre de 1979 , dans "Les parcours du savoir" 6 . Les liens entre les deux textes sont nombreux, et on peut passer facilement de l'un à l'autre - ce que je ne me priverai pas de faire - puisque c'est la même question qui est posée dans les deux cas: qu'est-ce que la scientificité? Sans faire des propositions de Greimas des références obligées, j'utiliserai tout de même l'effort de réflexion qu'elles représentent comme repère, afin d'indiquer sur quelques points comment elles peuvent éclairer, par analogie, la question du littéraire et de sa lecture.

4 Car pour les autres, les classiques déjà consacrés, Genette prétend assez brutalement que l'influence du lecteur n'a de toute façon aucune pertinence (p. 30).

5 Algirdas J. Greimas, Sémiotique et sciences sociales, Paris, Seuil, 1976, p. 7-42. L'abréviation SSS suivie de la page renverra à cette édition.

6 Algirdas J. Greimas et Éric Landowski, Introduction à l'analyse du discours en sciences sociales, Paris, Hachette, 1979, p. 5-28. L'abréviation INTR suivie de la page renverra à cette édition. 


\section{Les frontières}

Je passerai vite sur une question pourtant fascinante: les rapports entre le corpus qu'on choisit de lire et les propositions théoriques qui en sont les conséquences. Dans la pratique des textes scientifiques analysés qu'introduit Greimas, deux choses étonnent, et il faut lire cet étonnement. Tout d'abord, un point sur lequel je reviendrai un peu plus loin: ont été intentionnellement écartés les textes d'Althusser et de Lacan, considérés comme des "discours d'autorité [trop?] organisés autour de la relation quasiprophétique qui lie le discours de l'interprète à celui du fondateur" (INTR, 8), en l'occurrence Marx et Freud. L'autre surprise reliée au corpus marque un mouvement inverse qui a consisté, tout aussi intentionnellement, à inclure, pour l'étude du discours scientifique, deux discours non scientifiques, à savoir des discours à démarches purement interprétatives: une critique littéraire et un commentaire religieux.

Cette dernière procédure semble apporter un élément de réponse à mon interrogation de départ, laissant entendre qu'on peut appliquer à des textes non scientifiques des critères de lecture scientifiques, ici avec des objectifs heuristiques, "pour reconnaitre négativement, explique Greimas, quelques-unes des exigences du discours à vocation scientifique " (INTR, 8). J'enregistre le point sans le commenter, laissant ouverte la question de savoir si la lecture concomitante de discours non littéraires - ou la lecture intentionnellement non littéraire de la littérature - pourrait faire voir en creux certains caractères du discours ou de la lecture littéraires, dont elle fournirait une sorte de contre-exemple.

\section{Les références}

Mais ces questions de corpus cèdent vite le pas devant ce qui semble le caractère le plus contraignant et le plus fondamental de l'organisation du discours scientifique, à savoir toute la question de la référence, interne et externe, aussi dénommée anaphorisation (SSS, 23 et s.), soit le fait que le discours scientifique fonctionne à partir de deux lieux de référence dont il tire au fond toute sa véridiction, et qui sont situés ou bien dans le discours luimême (dans des expressions comme "l'on a vu que", "on verra que", qui renvoient les unes aux autres, [SSS, 23]), ou bien, et surtout, en dehors de lui: c'est le phénomène des renvois à des discours scientifiques déjà tenus, qui atteste ce que Greimas 
38

nomme une "permanence implicite du savoir du sujet portant sur le faire scientifique dans son ensemble" (SSS, 20-21). C'est dire que le sujet du discours scientifique ne joue en réalité "qu'un rôle d'agent médiateur car il fonde [lel savoir sur autre chose et sur un ailleurs, le relie, sous la forme d'une référence, à un autre discours ou à un autre système de savoir " (SSS, 21). Il y a donc toujours renvoi "d'un plan discursif qui dit la vérité à un autre qui lui sert de support. Et peu importe que ce second plan soit explicite ou qu'il reste implicite, il apparâit toujours comme un discours référentiel fondateur du discours véridique "(SSS, 26). C'est ici, à mon sens, qu'il faudrait situer la difficulté de prendre en considération les discours de Lacan et d'Althusser, dans lesquels le plan "qui dit la vérité" serait trop régulièrement en osmose par rapport à Freud et à Marx, et pas assez distinct des deux discours fondateurs.

La question de la référence impose d'emblée une transposition vers le discours littéraire et me ramène à mon sujet, la référence externe recouvrant bien évidemment, dans les discours qui nous intéressent, le travail de l'intertextualité, plus ou moins explicite, comme on le sait.

Mais c'est plutôt du côté de la référence interne, on s'en doute, et du fait justement qu'elle est à première vue moins évidente, qu'on aurait profit à raffiner davantage l'analyse; dans le discours scientifique, la référence interne supporte en effet directement le contenu du nouveau savoir produit, et qui sera intégré ou non dans les discours existants. On pourrait faire l'hypothèse que de la même manière, mais aussi autrement, le discours littéraire doit bien fonctionner lui aussi sur une référence interne qui lui serait spécifique, étant entendu qu'il ne s'agira pas cette fois de référence à un discours d'autorité, pour une entreprise de véridiction et de persuasion - ce qui est le cas du discours scientifique - mais d'une construction de langage, le discours littéraire - là-dessus Genette a raison - étant en un sens retourné surlui-même, c'est-à-dire sur le fait langagier. Toute la question est cependant de savoir où et comment lire, dans la syntagmatique d'un texte, et par exemple d'un roman, l'équivalent de cette anaphorisation interne que Greimas assimile, dans le discours scientifique, à la redondance d'expressions comme "il nous faut", "il est nécessaire ", "nous sommes obligés de reconnaître que ", "il est bon" (SSS, 34), toutes expressions qui, sortes de "présupposé permanent" (SSS, 34), surdéterminent et modalisent ce qu'elles encadrent, et construisent la science en opération de véridiction. 
C'est certainement là qu'il faut situer la proposition de notre équipe de recherche. Le plan de la référence interne, qui encadre et qui modalise ce que nous pourrions appeler le contenu du discours littéraire (dans le cas du roman, son anecdote), serait manifesté par le système des figures de l'écrit. Immanquablement entremêlées à l'anecdote, ces figures en surdétermineraient le contenu, au sens où elles seraient déterminantes pour sa signification. L'anecdote d'un roman ne serait validée - et pour reprendre la comparaison du discours scientifique, rendue véridictoire - qu'en fonction des figures de l'écrit et dans la direction indiquée par celles-ci. Ce n'est pas ici le lieu de préciser comment sont repérées les figures, ni comment elles sont articulées à l'anecdote: cela a été fait ailleurs ${ }^{7}$. Notre propos est de suggérer seulement qu'elles permettent une lecture littéraire.

On pense souvent que ce que j'ai appelé ici, après Greimas, la référence externe dans les textes littéraires, facile à repérer puisqu'il s'agit d'allusions à des discours littéraires déjà tenus, constituerait une garantie presque absolue d'authenticité littéraire. C'est pourquoi, et c'est là un phénomène de lecture, un texte rempli de rêférences littéraires, qu'il soit ou non de fiction, a toutes les chances d'être perçu et lu comme un texte appartenant de bon droit à la littérature.

Mais dans le cas du discours littéraire et du fait de son caractère fictif - contrairement au discours scientifique - , ce type de référence externe doit être saisi comme un processus superficiel, processus trompeur qui ne nous intéresse que dans la mesure où l'on comprend qu'il en cache, ou plutôt qu'il en simule un autre qui l'intègre, celui précisément de la référence interne, spécifique du travail de l'écriture, et dont on pourrait dire que la référence externe ne serait qu'une variante parmi d'autres.

C'est dire qu'au-delà du fait, par exemple, que Le vieux chagrin de Jacques Poulin soit parsemé de références à Hemingway, ou aux Mille et une nuits, encore faut-il que ces figures, et d'autres plus exclusivement internes au texte, comme les nombreux billets que le héros écrit à Marika, ou encore l'acte d'adoption final qu'il élabore sous l'oeil et sur les ordres de La Petite, soient lues dans

7 Voir * L'inscription de l'écriture dans Marie Calumet", Voix E images, $\mathrm{n}^{\circ} 46$ (automne 1990), p. 80-95, et dans l'Introduction métbodologique d'un ouvrage (en préparation) de notre groupe de recherche sur la question. 
40

leur fonction de cadre, nécessaire à la relativisation et à la surdétermination des diverses particularités de l'anecdote.

Quand Greimas pose un niveau déontique du discours scientifique, il s'attache à prouver que toutes les expressions anaphoriques qui émergent à la surface du discours sont formulées en termes de nécessité, et sont là "comme si une telle organisation syntaxique, et pas une autre, était indispensable, comme si un tel parcours devait être emprunté, qui, seul, permettait de mener à bien la tâche" (SSS, 35). Tout en émettant quelques réserves quant au caractère anthropomorphe avec lequel sont décrits ici le faire et le projet scientifiques, on pourrait dire que les figures de l'écrit, dans un texte littéraire, quelle que soit l'ampleur de leur déploiement, sont ce qui constitue ce plan nécessaire de référence, ce niveau déontique essentiel au projet proprement "scriptural" de ce discours.

Ma conclusion sera double. A priori, il ne semble pas que la littérarité puisse être attribuée, comme les classifications permissives de Genette autorisent, je pense, à le faire, à n'importe quel texte de non-fiction, voire à un éventail indéterminé de ces textes. Le texte littéraire est celui dont il peut être démontré qu'il fonctionne sur une anaphorisation interne qui se répercute, se bute et s'achève sur de l'écrit. C'est-à-dire que ce texte reprend, par les diverses figures de l'écrit entremêlées à son contenu, fictif ou pas, un jeu de va-et-vient qui s'appuie, dans le discours scientifique, s'agissant de persuasion, sur le discours d'autorité, et qui ici, où il s'agit ultimement d'écrire, s'ancre dans de l'écrit.

Il est bien entendu que d'un point de vue sociologique ouvert, n'importe quel lecteur peut décider de la nature de la littérature et du caractère littéraire ou pas de sa lecture. On aura compris que la perspective théorique sur la question est forcément plus étroite, et que littéraire s'y entend alors au sens restreint où il est l'adjectif de littérarité. Dans cette optique, la lecture spécifiquement littéraire des textes serait celle qui perçoit et démonte une organisation textuelle, en arrivant à privilégier une problématique de lecture où c'est de toutes les façons l'écrit qui, au sens fort, donne la signification. 